\title{
Effective collision strengths for transitions in Fe XIII ${ }^{\star}$
}

\author{
K. M. Aggarwal and F. P. Keenan
}

\begin{abstract}
Department of Pure and Applied Physics, The Queen's University of Belfast, Belfast BT7 1NN, Northern Ireland, UK e-mail: K. Aggarwal@qub.ac.uk
\end{abstract}

Received 13 August 2004 / Accepted 13 September 2004

\begin{abstract}
Effective collision strengths for transitions among the lowest 97 fine-structure levels belonging to the $\left(1 s^{2} 2 s^{2} 2 p^{6}\right)$ $3 s^{2} 3 p^{2}, 3 s 3 p^{3}, 3 s^{2} 3 p 3 d, 3 p^{4}, 3 s 3 p^{2} 3 d$ and $3 s^{2} 3 d^{2}$ configurations of Fe XIII have been calculated using the fully relativistic Dirac Atomic $R$-matrix Code (DARC) of Norrington \& Grant (2004). Resonances have been resolved in the threshold region, and results are reported over a wide electron temperature range up to $\log T_{\mathrm{e}}=6.8 \mathrm{~K}$. Comparisons are made with the earlier available $R$-matrix results of Gupta \& Tayal (1998), and the accuracy of the data is assessed.
\end{abstract}

Key words. atomic data - atomic processes

\section{Introduction}

This is a continuation of our work on generating atomic data (namely energy levels, radiative rates, life-times, electron impact excitation collision strengths, and rate coefficients) for iron ions, as this is an abundant element, particularly in solar and fusion plasmas, and its emission lines are observed in almost all ionization stages. For the diagnostics and modelling of a variety of plasmas, theoretical atomic data are required, because corresponding results from experiments are often not available. In this work, we have already reported atomic data for Fe X (Aggarwal \& Keenan 2004b), Fe XI (Aggarwal \& Keenan 2003a,b), Fe XV (Aggarwal et al. 2003a), Fe XVII (Aggarwal et al. 2003b, 2004), Fe XVIII (Jonauskas et al. 2004), Fe XXI (Aggarwal \& Keenan 2001), and Fe XXIV (McKeown et al. 2004), and in this paper we present our results of excitation rate coefficients for transitions among the $\left(1 s^{2} 2 s^{2} 2 p^{6}\right) 3 s^{2} 3 p^{2}, 3 s 3 p^{3}, 3 s^{2} 3 p 3 d, 3 p^{4}, 3 s 3 p^{2} 3 d$ and $3 s^{2} 3 d^{2}$ configurations of Fe XIII. The corresponding results for other atomic parameters for this ion have already been reported in our recent paper (Aggarwal \& Keenan 2004a).

Emission lines of Fe XIII have particularly been observed in the ultraviolet (UV: 900-2000 A) and extreme ultraviolet (EUV: 150-900 ̊) range of the solar spectrum (Malinovsky \& Heroux 1973; Dere 1978, 1982; Dere et al. 1979; Feldman 1981; Thomas \& Neupert 1994), as well as in late-type stars (Drake et al. 1995; Laming et al. 1995; Fuhrmeister et al. 2004). Many of its lines from quiet and active solar regions, including flares, have been observed (Brosius et al. 1996, 1998, 2000) by Skylab, the Solar EUV Research Telescope and Spectrograph (SERTS), and the Coronal Diagnostic Spectrometer (CDS) on

^ Tables 2 and 3 are available only in electronic form at the CDS via anonymous ftp to cdsarc.u-strasbg.fr (130.79.128.5) or via http: //cdsweb.u-strasbg.fr/cgi-bin/qcat? J/A+A/429/1117 board the Solar and Heliospheric Observatory (SOHO). Almost all observed Fe XIII emission lines, whether in the Sun or other late-type stars (for example: Alpha Cen and Procyon), have proved excellent tools for density diagnostics, particularly at coronal temperatures (Keenan et al. 1995). Additionally, transitions within the $3 \mathrm{~s}^{2} 3 \mathrm{p}^{2}$ ground configuration give rise to prominent lines in the optical and near infrared, and have proved to be useful density indicators for ground-based observers (Young 2004). Therefore, Fe XIII is one of the prominent iron ion, for which theoretical atomic data are of vital importance for understanding plasma characteristics.

Earlier calculations for Fe XIII have been performed by many workers, and among the most prominent results available today are the calculations of Fawcett \& Mason (1989, henceforth FM) and Gupta \& Tayal (1998, henceforth GT). FM adopted the SuperStructure (SS) program of Eissner et al. (1974) for the construction of their wavefunctions, and values of collision strengths $(\Omega)$ were computed from the Distorted-Wave (DW) program of Eissner \& Seaton (1972). They included configuration interaction (CI) among six configurations (namely $3 \mathrm{~s}^{2} 3 \mathrm{p}^{2}, 3 \mathrm{~s} 3 \mathrm{p}^{3}, 3 \mathrm{~s}^{2} 3 \mathrm{p} 3 \mathrm{~d}, 3 \mathrm{p}^{4}, 3 \mathrm{~s} 3 \mathrm{p}^{2} \mathrm{~d}^{2}$ and $3 p^{3} 3 \mathrm{~d}$ ), but reported energy levels and electron impact collision strengths for transitions among the lowest 27 levels of the $3 \mathrm{~s}^{2} 3 \mathrm{p}^{2}, 3 \mathrm{~s} 3 \mathrm{p}^{3}$ and $3 \mathrm{~s}^{2} 3 \mathrm{p} 3 \mathrm{~d}$ configurations only. Their calculations for $\Omega$ were in $L S$ coupling, and results in $L S J$ coupling were obtained through the JAJOM program of Saraph (1978). Furthermore, one-body relativistic operators were included through term coupling coefficients (TCC). Values of $\Omega$ were reported at three energies well above thresholds, i.e. at 15, 30 and $45 \mathrm{Ryd}$, and for transitions among the levels of the $3 \mathrm{~s}^{2} 3 \mathrm{p}^{2}$ ground configuration, and from these levels to higher excited levels of the $3 \mathrm{~s} 3 \mathrm{p}^{3}$ and $3 \mathrm{~s}^{2} 3 \mathrm{p} 3 \mathrm{~d}$ configurations. Therefore, apart from an incomplete set of results, the main deficiency of their calculations is the lack of resonance structure, 
which significantly affects the calculations of effective collision strengths $(\Upsilon)$, particularly for the forbidden transitions.

The above deficiency of the FM work was removed by GT, who reported results for energy levels, radiative rates, collision strengths $(\Omega)$, and effective collision strengths $(\Upsilon)$ for transitions among 26 fine-structure levels of the $3 \mathrm{~s}^{2} 3 \mathrm{p}^{2}, 3 \mathrm{~s} 3 \mathrm{p}^{3}$ and $3 s^{2} 3 p 3 d$ configurations. For their calculations, they adopted the CIV3 program of Hibbert (1978) for the generation of wavefunctions, and the $R$-matrix code of Scott \& Taylor (1982) for computing $\Omega$. Furthermore, they included CI and one-body relativistic operators (in the Breit-Pauli approximation) in the determination of wavefunctions, and also resolved resonances in the threshold region to calculate results for $\Upsilon$ at temperatures below $5 \times 10^{6} \mathrm{~K}$, sufficient for most applications in plasma diagnostics. Therefore, their results should be the most reliable available today. However, in a recent paper Landi (2002) has emphasized a need for fresh calculations (for both transition rates and collision rates), because electron densities derived from line ratios calculated from the atomic data of GT and FM differ by a factor of two, and hence imply different results for plasma parameters.

Therefore, our aim is to assess the accuracy of available atomic data, especially for $\Omega$ and $\Upsilon$, so that results can be applied with confidence for plasma diagnostics. To achieve our aim we have employed the GRASP (General-purpose Relativistic Atomic Structure Package) code of Dyall et al. (1989), and the DARC (Dirac Atomic $R$-matrix Code) of Norrington \& Grant (2004) for the computations of wavefunctions and $\Omega$, respectively. This is in contrast to the use of the CIV3 (Hibbert 1975) and Breit-Pauli $R$-matrix (Scott \& Taylor 1982) programs by GT, and SS (Eissner et al. 1974) and DW (Eissner \& Seaton 1972) codes by FM. Thus our calculations are fully relativistic in the $j j$ coupling scheme, in comparison to their semi-relativistic approach of the $L S J$ coupling scheme.

Beside the above reason, there is scope for an extension of the results of both Fawcett \& Mason (1989) and Gupta \& Tayal (1998). We achieve this by extending the calculations to 97 levels, hence including resonances arising from higher lying levels, mainly from the $3 p^{4}, 3 s 3 p^{2} 3 d$ and $3 \mathrm{~s}^{2} 3 \mathrm{~d}^{2}$ configurations.

\section{Calculations}

The $\left(1 s^{2} 2 s^{2} 2 p^{6}\right) \quad 3 s^{2} 3 p^{2}, \quad 3 s 3 p^{3}, \quad 3 s^{2} 3 p 3 d, \quad 3 p^{4}, \quad 3 s 3 p^{2} 3 d$ and $3 s^{2} 3 d^{2}$ configurations of Fe XIII give rise to 97 finestructure levels, listed in Table 1. To calculate level energies, oscillator strengths, and radiative rates for allowed transitions among these levels, we have adopted the GRASP code of Dyall et al. (1989). Thus relativistic effects have been fully taken into account, unlike earlier calculations which have neglected the two-body relativistic operators. Our results for energy levels and radiative rates have already been reported and compared in our previous paper (Aggarwal \& Keenan 2004a), and hence will not be discussed here again. However, to briefly recapitulate we may state here that our energy levels were assessed to be accurate to $\sim 5 \%$, and the radiative rates to $\sim 20 \%$, for a majority of the transitions. Our calculated energy levels, along with the available experimental compilations by NIST (http://www . physics.nist/gov/PhysRefData) and theoretical CIV3 results of GT, are listed in Table 1 for a ready reference. Also included in Table 1 are the life-times for all excited levels, which were not reported in our earlier publication. The corresponding life-times $(\tau)$ from experiments are not available for comparison, except for two levels, namely $3 \mathrm{~s}^{2} 3 \mathrm{p}^{2}{ }^{1} \mathrm{D}_{2}$ and $3 \mathrm{~s}^{2} 3 \mathrm{p} 3 \mathrm{~d}^{3} \mathrm{~F}_{4}^{\circ}$ (Träbert et al. 2003). However, we hope that the presently listed life-times for all levels will be useful for comparisons from future experiments.

In our earlier publication (Aggarwal \& Keenan 2004a), we reported radiative rates for allowed and intercombination (E1: electric dipole) transitions alone. However, we have now performed calculations for other types of transitions as well, i.e. electric quadrupole (E2), magnetic dipole (M1) and magnetic quadrupole (M2), because results for these transitions are also required in any diagnostics or modelling calculation (Del Zanna et al. 2004). Therefore, in Table 2 we report our radiative rates for transitions among the 97 levels of Fe XIII, which are a sum over all the above four types of transitions. We hope that the presently reported results, along with our excitation rates discussed below, will be highly useful for plasma diagnostics.

Details of our calculations for collision strengths have already been given in our earlier publication (Aggarwal \& Keenan 2004a). To recapitulate, we have employed the fully relativistic DARC program of Norrington \& Grant (2004), which is based on the $j j$ coupling scheme, and uses the DiracCoulomb Hamiltonian in the $R$ - matrix approach. The $R$-matrix radius was adopted to be $3.0 \mathrm{au}$, and 15 continuum orbitals were included for each channel angular momentum for the expansion of the wavefunction. This allowed us to compute $\Omega$ up to an energy of 120 Ryd. The maximum number of channels for a partial wave is 475 , and the corresponding size of the Hamiltonian matrix is 7138. In order to obtain convergence of $\Omega$ for all transitions and at all energies, we have included all partial waves with angular momentum $J \leq 39.5$, although a higher range would have been preferable for the convergence of allowed transitions, especially at higher energies. However, to account for the inclusion of higher neglected partial waves, we have included a top-up, based on the Coulomb-Bethe approximation for allowed transitions and geometric series for forbidden transitions.

In a detailed comparison in our earlier work (Aggarwal $\&$ Keenan 2004a), we demonstrated that the reported results of FM for $\Omega$ are deficient for almost all transitions. This is mainly because of their inclusion of a limited range of partial waves, with angular momentum of only $L \leq 7$. This limited range of partial waves is not sufficient for the convergence of $\Omega$ for forbidden transitions (see Figs. 1 and 2 of Aggarwal \& Keenan), and is inadequate for the inclusion of Coulomb-Bethe approximation for the higher neglected partial waves. As a result of this, their listed values of $\Omega$ are underestimated for the forbidden transitions and overestimated for the allowed transitions - see Table 4 of Aggarwal \& Keenan. Since FM did not report any results for $\Upsilon$, we will not discuss their calculations further. On the other hand, the reported results of GT for $\Omega$ are in broad agreement with our DARC calculations, although 
Table 1. Target levels of Fe XIII with their threshold energies (in Ryd) and life-times ( $\tau$ in s).

\begin{tabular}{|c|c|c|c|c|c|c|c|c|}
\hline Index & Configuration & Level & Expt. $^{a}$ & GRASP $^{b}$ & GRASP $^{c}$ & $\mathrm{CIV}^{d}$ & $\overline{\tau^{b}}$ & $\overline{\tau^{c}}$ \\
\hline $1 \ldots \ldots$ & $3 s^{2} 3 p^{2}$ & ${ }^{3} \mathrm{P}_{0}$ & 0.0000 & 0.0000 & 0.0000 & 0.0000 & & \\
\hline $2 \ldots \ldots$ & $3 s^{2} 3 p^{2}$ & ${ }^{3} \mathrm{P}_{1}$ & 0.0848 & 0.0835 & 0.0835 & 0.0768 & 7.479-02 & $7.505-02$ \\
\hline $3 \ldots \ldots$ & $3 s^{2} 3 p^{2}$ & ${ }^{3} \mathrm{P}_{2}$ & 0.1691 & 0.1700 & 0.1693 & 0.1582 & $9.398-02$ & $9.601-02$ \\
\hline $4 \ldots \ldots$. & $3 s^{2} 3 p^{2}$ & ${ }^{1} D_{2}$ & 0.4380 & 0.4629 & 0.4570 & 0.4366 & $6.043-03$ & $6.134-03$ \\
\hline $5 \ldots \ldots$ & $3 s^{2} 3 p^{2}$ & ${ }^{1} \mathrm{~S}_{0}$ & 0.8339 & 0.8497 & 0.8513 & 0.8612 & 8.807-04 & 8.789-04 \\
\hline $6 \ldots \ldots$ & $3 \mathrm{~s} 3 \mathrm{p}^{3}$ & ${ }^{5} \mathrm{~S}_{2}^{\circ}$ & & 1.9719 & 1.8987 & & $9.295-08$ & $8.680-08$ \\
\hline $7 \ldots \ldots$ & $3 \mathrm{~s} 3 \mathrm{p}^{3}$ & ${ }^{3} \mathrm{D}_{1}^{\circ}$ & 2.6172 & 2.7427 & 2.6004 & 2.5978 & $5.597-10$ & $6.316-10$ \\
\hline $8 \ldots \ldots$ & $3 \mathrm{~s} 3 \mathrm{p}^{3}$ & ${ }^{3} \mathrm{D}_{2}^{\circ}$ & 2.6186 & 2.7437 & 2.6017 & 2.5982 & $6.028-10$ & $6.847-10$ \\
\hline $9 \ldots \ldots$ & $3 \mathrm{~s} 3 \mathrm{p}^{3}$ & ${ }^{3} \mathrm{D}_{3}^{\circ}$ & 2.6445 & 2.7683 & 2.6271 & 2.6212 & $6.865-10$ & $7.822-10$ \\
\hline $10 \ldots \ldots$ & $3 \mathrm{~s} 3 \mathrm{p}^{3}$ & ${ }^{3} \mathrm{P}_{0}^{\circ}$ & & 3.1533 & 2.9915 & 2.9906 & $2.109-10$ & $2.404-10$ \\
\hline $11 \ldots \ldots$ & $3 \mathrm{~s} 3 \mathrm{p}^{3}$ & ${ }^{3} \mathrm{P}^{\circ}{ }_{1}$ & 3.0040 & 3.1595 & 2.9978 & 2.9956 & $2.144-10$ & $2.416-10$ \\
\hline $12 \ldots \ldots$ & $3 \mathrm{~s} 3 \mathrm{p}^{3}$ & ${ }^{3} \mathrm{P}_{2}^{\circ}$ & 3.0097 & 3.1649 & 3.0033 & 2.9910 & $2.375-10$ & $2.687-10$ \\
\hline $13 \ldots \ldots$ & $3 \mathrm{~s} 3 \mathrm{p}^{3}$ & ${ }^{1} \mathrm{D}_{2}^{\circ}$ & 3.3017 & 3.5222 & 3.3084 & 3.2968 & $1.698-10$ & $1.888-10$ \\
\hline $14 \ldots \ldots$ & $3 s^{2} 3 p 3 d$ & ${ }^{3} \mathrm{~F}_{2}^{\circ}$ & & 4.1285 & 3.9508 & 3.9906 & $2.120-09$ & $1.918-09$ \\
\hline $15 \ldots \ldots$ & $3 \mathrm{~s} 3 \mathrm{p}^{3}$ & ${ }^{3} \mathrm{~S}^{\circ}{ }_{1}$ & 3.7860 & 4.1498 & 3.8485 & 3.8390 & $1.650-11$ & $1.592-11$ \\
\hline $16 \ldots \ldots$ & $3 s^{2} 3 p 3 d$ & ${ }^{3} \mathrm{~F}_{3}^{\circ}$ & & 4.1901 & 4.0120 & 4.0500 & $3.290-09$ & $3.247-09$ \\
\hline $17 \ldots \ldots$ & $3 s^{2} 3 p 3 d$ & ${ }^{3} \mathrm{~F}_{4}^{\circ}$ & & 4.2822 & 4.1025 & 4.1366 & $7.745-03$ & $8.152-03$ \\
\hline $18 \ldots \ldots$ & $3 \mathrm{~s} 3 \mathrm{p}^{3}$ & ${ }^{1} \mathrm{P}^{\circ}{ }_{1}$ & 3.9918 & 4.3144 & 4.0650 & 4.0348 & $1.524-11$ & $2.290-11$ \\
\hline $19 \ldots \ldots$ & $3 s^{2} 3 p 3 d$ & ${ }^{3} \mathrm{P}_{2}^{\circ}$ & 4.4320 & 4.7668 & 4.5045 & 4.4884 & $1.706-11$ & $1.853-11$ \\
\hline $20 \ldots \ldots$ & $3 s^{2} 3 p 3 d$ & ${ }^{3} \mathrm{P}^{\circ}{ }_{1}$ & 4.5102 & 4.8245 & 4.5788 & 4.5594 & $1.582-11$ & $1.794-11$ \\
\hline $21 \ldots \ldots$ & $3 s^{2} 3 p 3 d$ & ${ }^{3} \mathrm{P}_{0}^{\circ}$ & 4.5867 & 4.8765 & 4.6310 & 4.6076 & $1.790-11$ & $2.022-11$ \\
\hline $22 \ldots \ldots$ & $3 s^{2} 3 p 3 d$ & ${ }^{1} \mathrm{D}_{2}^{\circ}$ & 4.5460 & 4.9284 & 4.6237 & 4.5960 & $1.258-11$ & $1.633-11$ \\
\hline $23 \ldots \ldots$ & $3 s^{2} 3 p 3 d$ & ${ }^{3} \mathrm{D}_{1}^{\circ}$ & 4.6156 & 4.9392 & 4.6924 & 4.6642 & $1.273-11$ & $1.446-11$ \\
\hline $24 \ldots \ldots$ & $3 s^{2} 3 p 3 d$ & ${ }^{3} \mathrm{D}_{2}^{\circ}$ & 4.6406 & 4.9689 & 4.7194 & 4.6918 & $1.290-11$ & $1.432-11$ \\
\hline $25 \ldots \ldots$ & $3 s^{2} 3 p 3 d$ & ${ }^{3} \mathrm{D}_{3}^{\circ}$ & 4.6400 & 4.9694 & 4.7219 & 4.6968 & $1.222-11$ & $1.393-11$ \\
\hline $26 \ldots \ldots$ & $3 s^{2} 3 p 3 d$ & ${ }^{1} \mathrm{~F}_{3}^{\circ}$ & 5.0745 & 5.4286 & 5.1884 & 5.2120 & $1.161-11$ & $1.294-11$ \\
\hline $27 \ldots \ldots$ & $3 p^{4}$ & ${ }^{3} \mathrm{P}_{2}$ & & 5.5487 & 5.5444 & & $1.326-10$ & $1.224-10$ \\
\hline $28 \ldots \ldots$ & $3 s^{2} 3 p 3 d$ & ${ }^{1} \mathrm{P}^{\circ}{ }_{1}$ & 5.2005 & 5.5975 & 5.3014 & 5.2694 & $1.303-11$ & $1.650-11$ \\
\hline $29 \ldots \ldots$ & $3 p^{4}$ & ${ }^{3} \mathrm{P}_{1}$ & & 5.6581 & 5.6547 & & $1.293-10$ & $1.193-10$ \\
\hline $30 \ldots \ldots$ & $3 p^{4}$ & ${ }^{3} \mathrm{P}_{0}$ & & 5.6877 & 5.6844 & & $1.248-10$ & $1.156-10$ \\
\hline $31 \ldots \ldots$ & $3 \mathrm{~s} 3 \mathrm{p}^{2} 3 \mathrm{~d}$ & ${ }^{5} \mathrm{~F}_{1}$ & & 5.7727 & 5.7591 & & $1.464-08$ & $1.259-08$ \\
\hline $32 \ldots \ldots$ & $3 \mathrm{~s} 3 \mathrm{p}^{2} 3 \mathrm{~d}$ & ${ }^{5} \mathrm{~F}_{2}$ & & 5.7924 & 5.7792 & & 4.381-09 & 5.591-09 \\
\hline $33 \ldots \ldots$ & $3 p^{4}$ & ${ }^{1} \mathrm{D}_{2}$ & & 5.7955 & 5.7830 & & $2.082-10$ & $1.776-10$ \\
\hline $34 \ldots \ldots$ & $3 \mathrm{~s} 3 \mathrm{p}^{2} 3 \mathrm{~d}$ & ${ }^{5} \mathrm{~F}_{3}$ & & 5.8246 & 5.8116 & & $2.687-08$ & $2.500-08$ \\
\hline $35 \ldots \ldots$ & $3 \mathrm{~s} 3 \mathrm{p}^{2} 3 \mathrm{~d}$ & ${ }^{5} \mathrm{~F}_{4}$ & & 5.8698 & 5.8569 & & $1.284-07$ & $1.202-07$ \\
\hline $36 \ldots \ldots$ & $3 \mathrm{~s} 3 \mathrm{p}^{2} 3 \mathrm{~d}$ & ${ }^{5} \mathrm{~F}_{5}$ & & 5.9286 & 5.9151 & & $1.078-07$ & $1.269-07$ \\
\hline $37 \ldots \ldots$ & $3 \mathrm{~s} 3 \mathrm{p}^{2} 3 \mathrm{~d}$ & ${ }^{5} \mathrm{D}_{0}$ & & 5.9856 & 5.9873 & & $3.072-09$ & $3.266-09$ \\
\hline $38 \ldots \ldots$ & $3 \mathrm{~s} 3 \mathrm{p}^{2} 3 \mathrm{~d}$ & ${ }^{5} \mathrm{D}_{1}$ & & 5.9911 & 5.9924 & & 2.464-09 & $2.487-09$ \\
\hline $39 \ldots \ldots$ & $3 \mathrm{~s} 3 \mathrm{p}^{2} 3 \mathrm{~d}$ & ${ }^{5} \mathrm{D}_{2}$ & & 6.0016 & 6.0024 & & $1.797-09$ & $1.711-09$ \\
\hline $40 \ldots \ldots$ & $3 \mathrm{~s} 3 \mathrm{p}^{2} 3 \mathrm{~d}$ & ${ }^{5} \mathrm{D}_{3}$ & & 6.0179 & 6.0183 & & $1.612-09$ & $1.444-09$ \\
\hline $41 \ldots \ldots$ & $3 \mathrm{~s} 3 \mathrm{p}^{2} 3 \mathrm{~d}$ & ${ }^{5} \mathrm{D}_{4}$ & & 6.0444 & 6.0456 & & $4.472-08$ & $3.703-08$ \\
\hline $42 \ldots \ldots$ & $3 \mathrm{~s} 3 \mathrm{p}^{2}\left({ }^{1} \mathrm{D}\right) 3 \mathrm{~d}$ & ${ }^{3} F_{2}$ & & 6.2614 & 6.2600 & & $1.310-09$ & $1.270-09$ \\
\hline $43 \ldots \ldots$ & $3 \mathrm{~s} 3 \mathrm{p}^{2}\left({ }^{1} \mathrm{D}\right) 3 \mathrm{~d}$ & ${ }^{3} \mathrm{~F}_{3}$ & & 6.3008 & 6.2996 & & $1.387-09$ & $1.280-09$ \\
\hline $44 \ldots \ldots$ & $3 \mathrm{~s} 3 \mathrm{p}^{2}\left({ }^{1} \mathrm{D}\right) 3 \mathrm{~d}$ & ${ }^{3} \mathrm{~F}_{4}$ & & 6.3595 & 6.3587 & & 2.038-09 & $1.853-09$ \\
\hline $45 \ldots \ldots$ & $3 \mathrm{~s} 3 \mathrm{p}^{2} 3 \mathrm{~d}$ & ${ }^{5} \mathrm{P}_{3}$ & & 6.4703 & 6.4298 & & $1.464-11$ & $1.669-11$ \\
\hline $46 \ldots \ldots$ & $3 \mathrm{~s} 3 \mathrm{p}^{2} 3 \mathrm{~d}$ & ${ }^{5} \mathrm{P}_{2}$ & & 6.5030 & 6.4626 & & $1.432-11$ & $1.629-11$ \\
\hline $47 \ldots \ldots$ & $3 \mathrm{~s} 3 \mathrm{p}^{2} 3 \mathrm{~d}$ & ${ }^{5} \mathrm{P}_{1}$ & & 6.5227 & 6.4821 & & $1.406-11$ & $1.599-11$ \\
\hline $48 \ldots \ldots$ & $3 p^{4}$ & ${ }^{1} \mathrm{~S}_{0}$ & & 6.6097 & 6.6104 & & $9.492-11$ & $9.222-11$ \\
\hline $49 \ldots \ldots$ & $3 \mathrm{~s} 3 \mathrm{p}^{2}\left({ }^{3} \mathrm{P}\right)\left({ }^{4} \mathrm{P}\right) 3 \mathrm{~d}$ & ${ }^{3} \mathrm{P}_{2}$ & & 6.7648 & 6.7631 & & $1.758-11$ & $2.015-11$ \\
\hline $50 \ldots \ldots$ & $3 \mathrm{~s} 3 \mathrm{p}^{2}\left({ }^{3} \mathrm{P}\right)\left({ }^{4} \mathrm{P}\right) 3 \mathrm{~d}$ & ${ }^{3} \mathrm{P}_{1}$ & & 6.8482 & 6.8462 & & $1.739-11$ & $1.985-11$ \\
\hline $51 \ldots \ldots$ & $3 \mathrm{~s} 3 \mathrm{p}^{2}\left({ }^{3} \mathrm{P}\right)\left({ }^{4} \mathrm{P}\right) 3 \mathrm{~d}$ & ${ }^{3} \mathrm{P}_{0}$ & & 6.9048 & 6.9047 & & $1.777-11$ & $2.016-11$ \\
\hline $52 \ldots \ldots$ & $3 \mathrm{~s} 3 \mathrm{p}^{2} 3 \mathrm{~d}$ & ${ }^{3} \mathrm{G}_{3}$ & & 7.0072 & 6.9720 & & 9.153-11 & $1.156-10$ \\
\hline
\end{tabular}


Table 1. continued.

\begin{tabular}{|c|c|c|c|c|c|c|c|c|}
\hline Index & Configuration & Level & Expt. $^{a}$ & GRASP $^{b}$ & GRASP $^{c}$ & $\mathrm{CIV}^{d}$ & $\overline{\overline{\tau^{b}}}$ & $\overline{\tau^{c}}$ \\
\hline $53 \ldots \ldots$ & $3 \mathrm{~s} 3 \mathrm{p}^{2} 3 \mathrm{~d}$ & ${ }^{3} \mathrm{G}_{4}$ & & 7.0220 & 6.9868 & & $9.985-11$ & $1.252-10$ \\
\hline $54 \ldots \ldots$ & $3 \mathrm{~s} 3 \mathrm{p}^{2} 3 \mathrm{~d}$ & ${ }^{3} \mathrm{G}_{5}$ & & 7.0414 & 7.0060 & & $1.089-10$ & $1.372-10$ \\
\hline $55 \ldots \ldots$ & $3 \mathrm{~s} 3 \mathrm{p}^{2}\left({ }^{3} \mathrm{P}\right)\left({ }^{4} \mathrm{P}\right) 3 \mathrm{~d}$ & ${ }^{3} \mathrm{D}_{1}$ & & 7.0666 & 7.0097 & & $2.613-11$ & $2.598-11$ \\
\hline $56 \ldots \ldots$ & $3 s 3 p^{2}\left({ }^{3} P\right)\left({ }^{4} P\right) 3 d$ & ${ }^{3} \mathrm{D}_{2}$ & & 7.0752 & 7.0186 & & $2.473-11$ & $2.514-11$ \\
\hline $57 \ldots \ldots$ & $3 s 3 p^{2}\left({ }^{3} P\right)\left({ }^{4} P\right) 3 d$ & ${ }^{3} \mathrm{D}_{3}$ & & 7.0921 & 7.0339 & & $2.250-11$ & $2.329-11$ \\
\hline $58 \ldots \ldots$ & $3 \mathrm{~s} 3 \mathrm{p}^{2} 3 \mathrm{~d}$ & ${ }^{1} \mathrm{G}_{4}$ & & 7.1333 & 7.1225 & & $4.598-10$ & $4.727-10$ \\
\hline $59 \ldots \ldots$ & $3 \mathrm{~s} 3 \mathrm{p}^{2}\left({ }^{1} \mathrm{D}\right) 3 \mathrm{~d}$ & ${ }^{1} \mathrm{~F}_{3}$ & & 7.2445 & 7.2348 & & $3.146-11$ & $3.946-11$ \\
\hline $60 \ldots \ldots$ & $3 s 3 p^{2}\left({ }^{3} P\right)\left({ }^{4} P\right) 3 d$ & ${ }^{3} \mathrm{~F}_{2}$ & & 7.3369 & 7.3136 & & $1.493-11$ & $1.746-11$ \\
\hline $61 \ldots \ldots$ & $3 s 3 p^{2}\left({ }^{3} P\right)\left({ }^{4} P\right) 3 d$ & ${ }^{3} \mathrm{~F}_{3}$ & & 7.3838 & 7.3622 & & $1.444-11$ & $1.674-11$ \\
\hline $62 \ldots \ldots$ & $3 \mathrm{~s} 3 \mathrm{p}^{2}\left({ }^{1} \mathrm{D}\right) 3 \mathrm{~d}$ & ${ }^{3} \mathrm{D}_{1}$ & & 7.3972 & 7.3870 & & $1.789-11$ & $2.243-11$ \\
\hline $63 \ldots \ldots$ & $3 \mathrm{~s} 3 \mathrm{p}^{2}\left({ }^{1} \mathrm{D}\right) 3 \mathrm{~d}$ & ${ }^{3} \mathrm{D}_{2}$ & & 7.4209 & 7.4109 & & $1.935-11$ & $2.392-11$ \\
\hline $64 \ldots \ldots$ & $3 s 3 p^{2}\left({ }^{3} P\right)\left({ }^{4} P\right) 3 d$ & ${ }^{3} \mathrm{~F}_{4}$ & & 7.4509 & 7.4280 & & $1.382-11$ & $1.581-11$ \\
\hline $65 \ldots \ldots$ & $3 \mathrm{~s} 3 \mathrm{p}^{2}\left({ }^{1} \mathrm{D}\right) 3 \mathrm{~d}$ & ${ }^{3} \mathrm{P}_{0}$ & & 7.4676 & 7.4415 & & $1.653-11$ & $1.778-11$ \\
\hline $66 \ldots \ldots$ & $3 \mathrm{~s} 3 \mathrm{p}^{2}\left({ }^{1} \mathrm{D}\right) 3 \mathrm{~d}$ & ${ }^{3} \mathrm{D}_{3}$ & & 7.4687 & 7.4609 & & $2.296-11$ & $2.887-11$ \\
\hline $67 \ldots \ldots$. & $3 \mathrm{~s} 3 \mathrm{p}^{2}\left({ }^{1} \mathrm{D}\right) 3 \mathrm{~d}$ & ${ }^{3} \mathrm{P}_{1}$ & & 7.4842 & 7.4587 & & $1.682-11$ & $1.798-11$ \\
\hline $68 \ldots \ldots$ & $3 \mathrm{~s} 3 \mathrm{p}^{2}\left({ }^{1} \mathrm{D}\right) 3 \mathrm{~d}$ & ${ }^{3} \mathrm{P}_{2}$ & & 7.5160 & 7.4910 & & $1.683-11$ & $1.790-11$ \\
\hline $69 \ldots \ldots$ & $3 \mathrm{~s} 3 \mathrm{p}^{2}\left({ }^{3} \mathrm{P}\right) 3 \mathrm{~d}$ & ${ }^{1} \mathrm{P}_{1}$ & & 7.5402 & 7.5333 & & $1.666-11$ & $1.759-11$ \\
\hline $70 \ldots \ldots$ & $3 \mathrm{~s} 3 \mathrm{p}^{2} 3 \mathrm{~d}$ & ${ }^{3} \mathrm{~S}_{1}$ & & 7.6629 & 7.6561 & & $1.610-11$ & $1.800-11$ \\
\hline $71 \ldots \ldots$ & $3 \mathrm{~s} 3 \mathrm{p}^{2}\left({ }^{1} \mathrm{~S}\right) 3 \mathrm{~d}$ & ${ }^{3} \mathrm{D}_{1}$ & & 7.7297 & 7.6830 & & $1.756-11$ & $1.822-11$ \\
\hline $72 \ldots \ldots$ & $3 \mathrm{~s} 3 \mathrm{p}^{2}\left({ }^{1} \mathrm{~S}\right) 3 \mathrm{~d}$ & ${ }^{3} \mathrm{D}_{2}$ & & 7.7356 & 7.6721 & & $1.860-11$ & $1.909-11$ \\
\hline $73 \ldots \ldots$ & $3 \mathrm{~s} 3 \mathrm{p}^{2}\left({ }^{1} \mathrm{~S}\right) 3 \mathrm{~d}$ & ${ }^{3} \mathrm{D}_{3}$ & & 7.7962 & 7.7111 & & $1.696-11$ & $1.750-11$ \\
\hline $74 \ldots \ldots$ & $3 \mathrm{~s} 3 \mathrm{p}^{2}\left({ }^{3} \mathrm{P}\right)\left({ }^{2} \mathrm{P}\right) 3 \mathrm{~d}$ & ${ }^{3} \mathrm{~F}_{2}$ & & 7.8615 & 7.8205 & & $1.338-11$ & $1.531-11$ \\
\hline $75 \ldots \ldots$ & $3 \mathrm{~s} 3 \mathrm{p}^{2}\left({ }^{3} \mathrm{P}\right)\left({ }^{2} \mathrm{P}\right) 3 \mathrm{~d}$ & ${ }^{3} \mathrm{~F}_{3}$ & & 7.9025 & 7.8650 & & $1.390-11$ & $1.550-11$ \\
\hline $76 \ldots \ldots$ & $3 \mathrm{~s} 3 \mathrm{p}^{2}\left({ }^{3} \mathrm{P}\right)\left({ }^{2} \mathrm{P}\right) 3 \mathrm{~d}$ & ${ }^{3} \mathrm{~F}_{4}$ & & 7.9385 & 7.8993 & & $1.399-11$ & $1.606-11$ \\
\hline $77 \ldots \ldots$ & $3 \mathrm{~s} 3 \mathrm{p}^{2}\left({ }^{1} \mathrm{D}\right) 3 \mathrm{~d}$ & ${ }^{1} \mathrm{D}_{2}$ & & 7.9594 & 7.9241 & & $2.016-11$ & $2.471-11$ \\
\hline $78 \ldots \ldots$ & $3 \mathrm{~s} 3 \mathrm{p}^{2}\left({ }^{3} \mathrm{P}\right)\left({ }^{2} \mathrm{P}\right) 3 \mathrm{~d}$ & ${ }^{3} \mathrm{D}_{3}$ & & 8.1752 & 8.1095 & & $1.252-11$ & $1.384-11$ \\
\hline $79 \ldots \ldots$ & $3 \mathrm{~s} 3 \mathrm{p}^{2}\left({ }^{3} \mathrm{P}\right)\left({ }^{2} \mathrm{P}\right) 3 \mathrm{~d}$ & ${ }^{3} \mathrm{D}_{2}$ & & 8.2051 & 8.1212 & & $1.213-11$ & $1.351-11$ \\
\hline $80 \ldots \ldots$ & $3 \mathrm{~s} 3 \mathrm{p}^{2}\left({ }^{3} \mathrm{P}\right)\left({ }^{2} \mathrm{P}\right) 3 \mathrm{~d}$ & ${ }^{3} \mathrm{D}_{1}$ & & 8.2295 & 8.1344 & & $1.239-11$ & $1.359-11$ \\
\hline $81 \ldots \ldots$ & $3 \mathrm{~s} 3 \mathrm{p}^{2}\left({ }^{1} \mathrm{~S}\right) 3 \mathrm{~d}$ & ${ }^{1} \mathrm{D}_{2}$ & & 8.2398 & 8.1926 & & $1.278-11$ & $1.266-11$ \\
\hline $82 \ldots \ldots$ & $3 \mathrm{~s} 3 \mathrm{p}^{2}\left({ }^{1} \mathrm{D}\right) 3 \mathrm{~d}$ & ${ }^{1} \mathrm{P}_{1}$ & & 8.3951 & 8.3257 & & $1.039-11$ & $1.112-11$ \\
\hline $83 \ldots \ldots$ & $3 s 3 p^{2} 3 d$ & ${ }^{1} \mathrm{~S}_{0}$ & & 8.3971 & 8.3913 & & $1.037-11$ & $1.086-11$ \\
\hline $84 \ldots \ldots$ & $3 \mathrm{~s} 3 \mathrm{p}^{2}\left({ }^{3} \mathrm{P}\right)\left({ }^{2} \mathrm{P}\right) 3 \mathrm{~d}$ & ${ }^{3} \mathrm{P}_{2}$ & & 8.6544 & 8.6114 & & $5.702-12$ & $6.700-12$ \\
\hline $85 \ldots \ldots$ & $3 \mathrm{~s} 3 \mathrm{p}^{2}\left({ }^{3} \mathrm{P}\right)\left({ }^{2} \mathrm{P}\right) 3 \mathrm{~d}$ & ${ }^{3} \mathrm{P}_{1}$ & & 8.7027 & 8.6585 & & $5.622-12$ & $6.589-12$ \\
\hline $86 \ldots \ldots$ & $3 \mathrm{~s} 3 \mathrm{p}^{2}\left({ }^{3} \mathrm{P}\right)\left({ }^{2} \mathrm{P}\right) 3 \mathrm{~d}$ & ${ }^{3} \mathrm{P}_{0}$ & & 8.7262 & 8.6850 & & $5.588-12$ & $6.592-12$ \\
\hline $87 \ldots \ldots$ & $3 \mathrm{~s} 3 \mathrm{p}^{2}\left({ }^{3} \mathrm{P}\right) 3 \mathrm{~d}$ & ${ }^{1} \mathrm{~F}_{3}$ & & 8.8997 & 8.8197 & & $6.851-12$ & $7.550-12$ \\
\hline $88 \ldots \ldots$ & $3 s^{2} 3 d^{2}$ & ${ }^{3} \mathrm{~F}_{2}$ & & 9.0282 & 8.9791 & & $8.305-12$ & $9.695-12$ \\
\hline $89 \ldots \ldots$ & $3 s^{2} 3 d^{2}$ & ${ }^{3} \mathrm{~F}_{3}$ & & 9.0382 & 8.9900 & & $8.557-12$ & $9.987-12$ \\
\hline $90 \ldots \ldots$ & $3 s^{2} 3 d^{2}$ & ${ }^{3} \mathrm{~F}_{4}$ & & 9.0472 & 9.0001 & & $8.834-12$ & $1.031-11$ \\
\hline $91 \ldots . .$. & $3 \mathrm{~s} 3 \mathrm{p}^{2}$ & ${ }^{1} \mathrm{D}_{2}$ & & 9.2874 & 9.1905 & & $5.295-12$ & 7.081-12 \\
\hline $92 \ldots \ldots$. & $3 s^{2} 3 d^{2}$ & ${ }^{1} \mathrm{G}_{4}$ & & 9.3583 & 9.2835 & & $1.263-11$ & $1.591-11$ \\
\hline $93 \ldots \ldots$. & $3 s^{2} 3 d^{2}$ & ${ }^{3} \mathrm{P}_{0}$ & & 9.3808 & 9.2843 & & $5.850-12$ & $7.453-12$ \\
\hline $94 \ldots \ldots$. & $3 s^{2} 3 d^{2}$ & ${ }^{3} \mathrm{P}_{1}$ & & 9.3883 & 9.2913 & & $5.883-12$ & $7.512-12$ \\
\hline $95 \ldots \ldots$ & $3 s^{2} 3 d^{2}$ & ${ }^{3} \mathrm{P}_{2}$ & & 9.4030 & 9.3049 & & $5.915-12$ & $7.588-12$ \\
\hline $96 \ldots \ldots$ & $3 s^{2} 3 d^{2}$ & ${ }^{1} \mathrm{D}_{2}$ & & 9.4997 & 9.4177 & & $5.957-12$ & $7.182-12$ \\
\hline $97 \ldots \ldots$. & $3 s^{2} 3 d^{2}$ & ${ }^{1} \mathrm{~S}_{0}$ & & 10.1460 & 10.1260 & & $4.809-12$ & $6.016-12$ \\
\hline
\end{tabular}

${ }^{a}$ NIST (http://www . physics.nist/gov/PhysRefData).

${ }^{b}$ Present results from 6 configurations and 97 levels - see Aggarwal \& Keenan (2004a).

${ }^{c}$ Present results from 13 configurations and 301 levels - see Aggarwal \& Keenan (2004a).

${ }^{d}$ Gupta \& Tayal (1998).

serious differences exist for a few forbidden transitions see, for example, the $\left(3 \mathrm{~s}^{2} 3 \mathrm{p}^{2}\right){ }^{3} \mathrm{P}_{0}-{ }^{1} \mathrm{~S}_{0}(1-5)$ transition in Table 4 of Aggarwal \& Keenan. This is mainly because of their adoption of the earlier version of the $R$-matrix program
(Scott \& Taylor 1982), which is known to have some errors - see Aggarwal \& Keenan for further details and comparisons. Nevertheless, it is not the results for $\Omega$, but for $\Upsilon$ which were applied by Landi (2002) in his detailed analysis of 
observational data for Fe XIII. Therefore, in the following section we focus our attention on the comparison of results for $\Upsilon$ between our calculations and those of GT.

Our calculated values of $\Omega$ have already been listed, compared, and discussed in detail in our previous paper (Aggarwal \& Keenan 2004a). However, in that paper we reported our results at only three energies of 15,30 and $45 \mathrm{Ryd}$, and for transitions from the lowest five levels to levels up to 28 , i.e. $3 \mathrm{~s}^{2} 3 \mathrm{p} 3 \mathrm{~d}{ }^{1} \mathrm{P}^{\circ}{ }_{1}$, because other results for comparison were available only for these transitions and energies. Therefore, in Table 2 we present our results of $\Omega$ for all transitions, and in a wider energy range up to 120 Ryd. The indices adopted to represent a transition are already given in Table 1 . These results of $\Omega$ are not directly applicable in any diagnostic or modelling work, but are very useful in assessing the accuracy of a calculation, and will be helpful for future comparisons.

\section{Effective collision strengths}

Effective collision strengths $\Upsilon$ are obtained after integrating $\Omega$ over a Maxwellian distribution of electron velocities, i.e.

$\Upsilon\left(T_{\mathrm{e}}\right)=\int_{0}^{\infty} \Omega(E) \exp \left(-E_{j} / k T_{\mathrm{e}}\right) \mathrm{d}\left(E_{j} / k T_{\mathrm{e}}\right)$,

where $\mathrm{E}_{j}$ is the incident energy of the electron with respect to the final state of the transition, $k$ is Boltzmann's constant, and $T_{\mathrm{e}}$ is the electron temperature in $\mathrm{K}$. Once the value of $\Upsilon$ is known for a transition, the corresponding value of the excitation $q(i, j)$ and de-excitation $q(j, i)$ rate coefficients can be easily obtained from the following simple relations:

$q(i, j)=\frac{8.63 \times 10^{-6}}{\omega_{i} T_{\mathrm{e}}^{1 / 2}} \Upsilon \exp \left(-E_{i j} / k T_{\mathrm{e}}\right) \quad \mathrm{cm}^{3} \mathrm{~s}^{-1}$

and

$q(j, i)=\frac{8.63 \times 10^{-6}}{\omega_{j} T_{\mathrm{e}}^{1 / 2}} \Upsilon \quad \mathrm{cm}^{3} \mathrm{~s}^{-1}$,

where $\omega_{i}$ and $\omega_{j}$ are the statistical weights of the initial $(i)$ and final $(j)$ states, respectively, and $\mathrm{E}_{i j}$ is the transition energy.

Since the threshold energy region is dominated by numerous resonances, $\Omega$ must be computed at a large number of energies in order to delineate these resonances. We have performed our calculations of $\Omega$ at over 6760 energies in the threshold region. Close to thresholds ( $\sim 0.1 \mathrm{Ryd}$ above a threshold) the energy mesh is $0.001 \mathrm{Ryd}$, and away from thresholds is 0.002 Ryd. Thus care has been taken to include as many resonances as possible, and with as fine a resolution as is computationally feasible. The values of $\Upsilon$ so computed are listed in Table 3 at a series of electron temperatures in the range $5.0 \leq \log T_{\mathrm{e}} \leq 6.8 \mathrm{~K}$, fully sufficient for the application of the data in solar, astrophysical and fusion plasmas.

As stated earlier, the only other results of $\Upsilon$ available for comparison are those of GT, who have reported results for a subset of transitions among the lowest 28 levels of Fe XIII, and in a temperature range of $5 \times 10^{5}$ to $5 \times 10^{6} \mathrm{~K}$. However, in a later paper Tayal (2000) has extended the lower range of electron temperature to $10000 \mathrm{~K}$, for transitions among the lowest

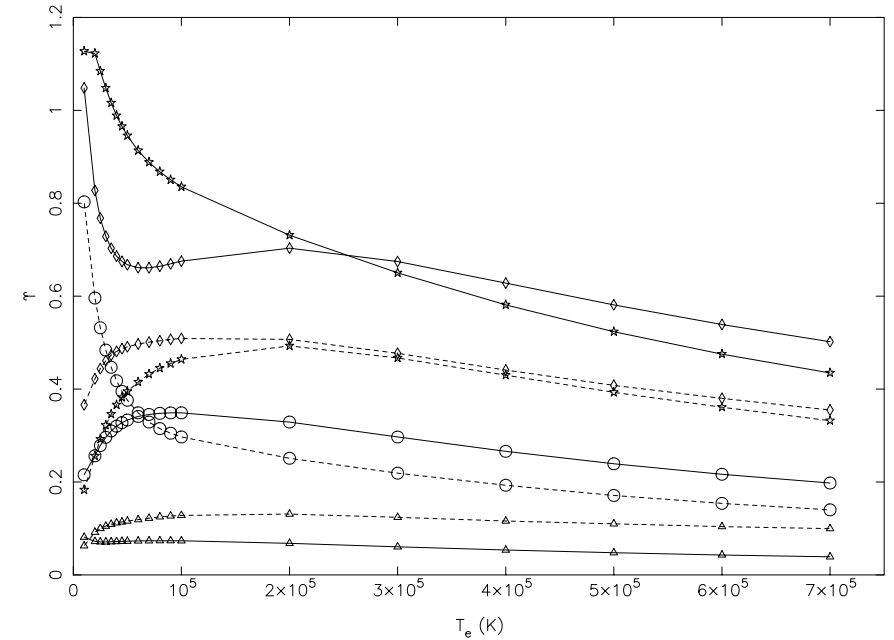

Fig. 1. Comparison of effective collision strengths for the 1-2 (stars), 1-3 (diamonds), 1-4 (circles) and 1-5 (triangles) transitions of Fe XIII. Continuous curve: present results, broken curve: results of Tayal (2000).

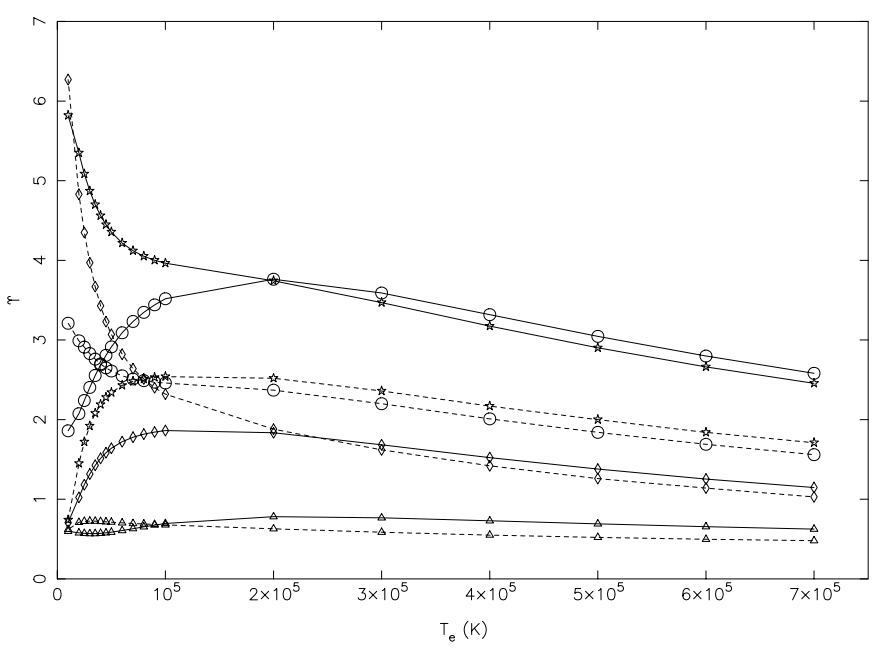

Fig. 2. Comparison of effective collision strengths for the 2-3 (stars), 2-4 (diamonds), 3-4 (circles) and 4-5 (triangles) transitions of Fe XIII. Continuous curve: present results, broken curve: results of Tayal (2000).

five levels of the $3 \mathrm{~s}^{2} 3 \mathrm{p}^{2}$ configuration. A comparison made between the two $R$-matrix calculations shows differences varying from a few percent to over an order of magnitude for a majority of the transitions, with the maximum discrepancy of a factor of 28 for the $3 s^{2} 3 p^{2}{ }^{3} P_{2}-3 s^{2} 3 p 3 d{ }^{1} D_{2}^{0}$ (3-28) allowed transition. We discuss these differences below.

In Figs. 1 and 2, we compare our values of $\Upsilon$ with those of Tayal (2000) for a few transitions among the lowest five levels of the $3 \mathrm{~s}^{2} 3 \mathrm{p}^{2}$ configuration at temperatures below $7 \times 10^{5} \mathrm{~K}$. As seen in these figures, there are two types of differences between the two sets of results. Firstly, there are transitions for which the differences are largest at the lowest temperature, and they decrease with increasing temperature. Example of such transitions are: ${ }^{3} \mathrm{P}_{0}-{ }^{3} \mathrm{P}_{1}(1-2),{ }^{3} \mathrm{P}_{0}-{ }^{3} \mathrm{P}_{2}(1-3),{ }^{3} \mathrm{P}_{0}-{ }^{1} \mathrm{D}_{2}$ (1-4), ${ }^{3} \mathrm{P}_{1}-{ }^{3} \mathrm{P}_{2}(2-3)$, and ${ }^{3} \mathrm{P}_{1}-{ }^{1} \mathrm{D}_{2}(2-4)$. For such transitions not only do the magnitudes of $\Upsilon$ differ by up to (almost) an 


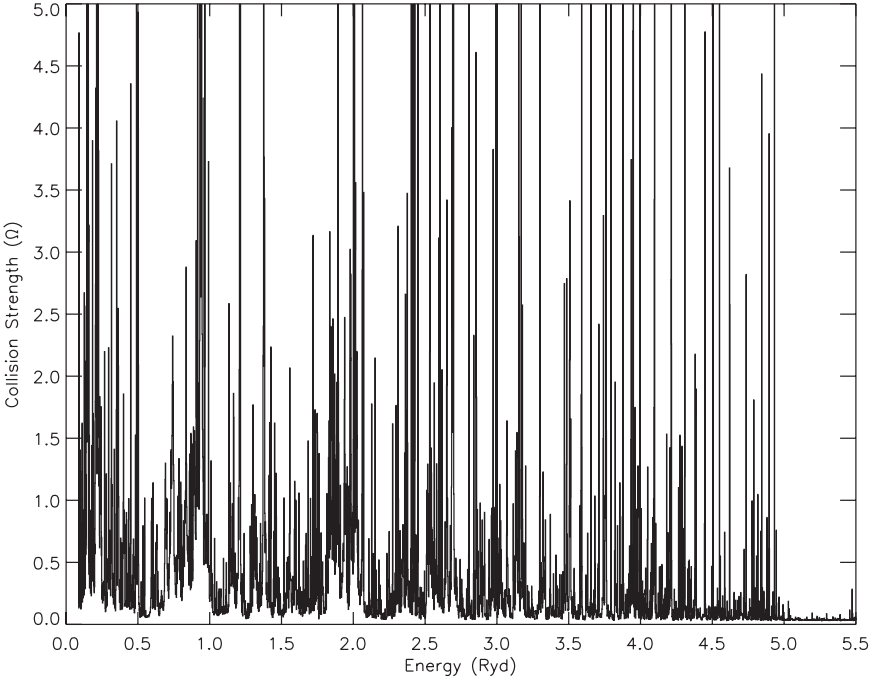

Fig. 3. Collision strengths for the $\left(3 \mathrm{~s}^{2} 3 \mathrm{p}^{2}\right){ }^{3} \mathrm{P}_{0}-{ }^{3} \mathrm{P}_{1}$ (1-2) transition of Fe XIII.

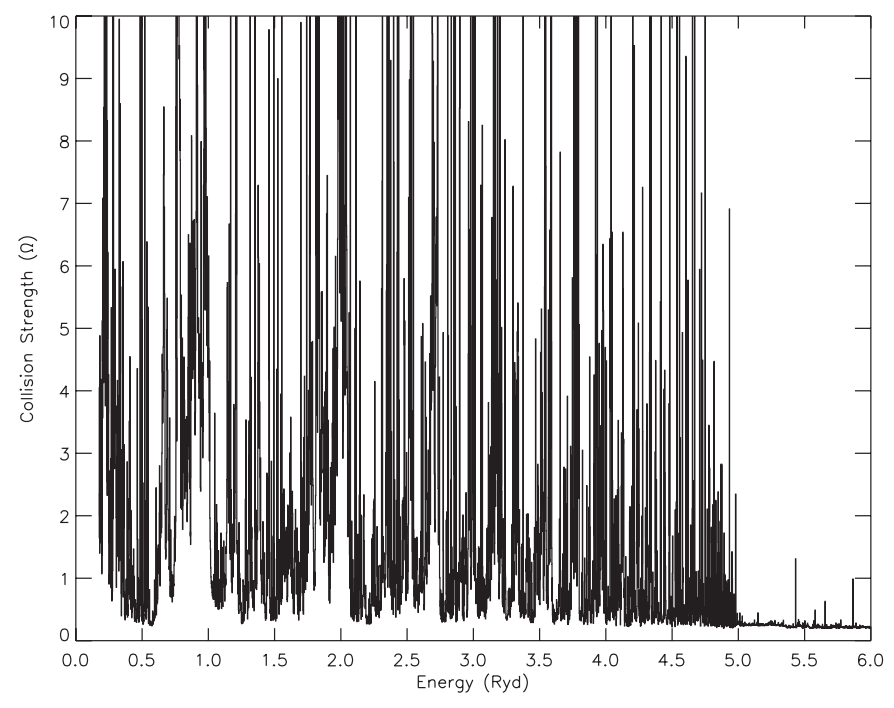

Fig. 4. Collision strengths for the $\left(3 \mathrm{~s}^{2} 3 \mathrm{p}^{2}\right){ }^{3} \mathrm{P}_{1}-{ }^{3} \mathrm{P}_{2}$ (2-3) transition of Fe XIII.

order of magnitude, but the trends with temperature are also opposite. This is the same type of problem as observed and discussed for transitions in Fe XI (Aggarwal \& Keenan 2003a), and arises because of the presence (or absence) of large resonances near the threshold. As examples, we show resonances for only two transitions, namely 1-2 and 2-3, in Figs. 3 and 4, respectively. For both of these transitions the resonances are not only dense and large, but arise from the threshold energies and cover a wide range up to $\sim 5$ Ryd. The presence of such resonances has clearly affected both the behaviour and magnitude of $\Upsilon$. Neither GT nor Tayal (2000) have shown resonances for these or any other transition in Fe XIII, and hence no direct comparisons can be made. However, it may be appropriate to emphasize here that there are three distinct differences between the two $R$-matrix calculations, which are mainly responsible for such large differences in the $\Upsilon$ results. Firstly, the energy mesh in our calculations is finer (better than 0.002 Ryd) in comparison to the coarse mesh of 0.005 Ryd of GT. This particularly affects the results when the magnitude of $\Omega$ is high for the near-threshold resonances, as is the case for the transitions under discussion. Secondly, our calculations are in the $j j$ coupling scheme in which fine-structure is included in the definition of channel coupling. This procedure accounts for the resonances more accurately in comparison to the $L S J$ coupling scheme adopted by GT. Finally, the calculations of GT are restricted to the lowest 28 levels, whereas our calculations include 97 levels. As a result of this, resonances arising from the higher levels are not included in their calculations, although this mainly affects the $\Upsilon$ results at higher temperatures. In conclusion, it should be clear that the present $R$-matrix calculations from DARC are comparatively more accurate than the semirelativistic $R$-matrix results of GT.

For some transitions, such as ${ }^{3} \mathrm{P}_{0}-{ }^{1} \mathrm{~S}_{0}$ (1-5) and ${ }^{1} \mathrm{D}_{2}-{ }^{1} \mathrm{~S}_{0}$ (4-5), differences in the two sets of $\Upsilon$ persist throughout the entire range of temperature. Some of these differences are due to the factors discussed above (particularly for the 4-5 transition), but are mainly because of the differences in the two sets of $\Omega$ (particularly for the 1-5 transition), as shown in Table 4 of Aggarwal \& Keenan (2004a) and discussed in detail in Sect. 4 of that paper. Since for many of the transitions for which the two sets of $\Omega$ are comparable, such as 1-2, 1-3 and 1-9, significant differences in $\Upsilon$ values persist up to the highest common temperature of $5 \times 10^{6} \mathrm{~K}$, we discuss the problem below in detail.

GT have reported their values of $\Upsilon$ up to a temperature of $5 \times 10^{6} \mathrm{~K}$, which amounts to $\sim 32$ Ryd in energy units. However, they have computed values of $\Omega$ up to an energy of 60 Ryd only, which is not sufficient for the convergence of the integral in Eq. (1), particularly for higher temperatures. As a result of this, their values of $\Upsilon$ are underestimated towards the higher end of the temperature range of their calculations. This can be easily verified by a closer look at their results of $\Omega$ in their Table 4 for transitions, such as 1-20, 2-22, 3-24, 426 and 5-28 (note the indices adopted are of those in Table 1 of the present paper), for which values of $\Omega$ increase with increasing energy (in both calculations), as these are allowed transitions. However, for all of these (and many more) transitions their corresponding results of $\Upsilon$ decrease with increasing temperature as seen in their Table 5 . To illustrate this point further, we focus on a single transition, namely $3 \mathrm{~s}^{2} 3 \mathrm{p}^{2}{ }^{3} \mathrm{P}_{1}-$ $3 s^{2} 3 p 3 d{ }^{3} D_{1}^{0}$ (2-23). In Fig. 5 we compare our results of $\Omega$ and $\Upsilon$ (in energy units) with those of GT. As expected, results for $\Omega$ increase with increasing energy in both sets of calculations, but the behaviour of $\Upsilon$ is exactly opposite. Our results for $\Upsilon$ increase with increasing temperature, whereas those of GT decrease. Additionally, there does not appear to be any relationship between their results of $\Omega$ and $\Upsilon$. Clearly, the $\Upsilon$ values reported by GT, and adopted by Landi (2002), are deficient for many transitions.

\section{Conclusions}

In this paper we have reported results for effective collision strengths for transitions among 97 fine-structure levels of the $3 s^{2} 3 p^{2}, 3 s 3 p^{3}, 3 s^{2} 3 p 3 d, 3 p^{4}, 3 s 3 p^{2} 3 d$ and $3 s^{2} 3 d^{2}$ configurations of Fe XIII. Relativistic effects and CI have been included 


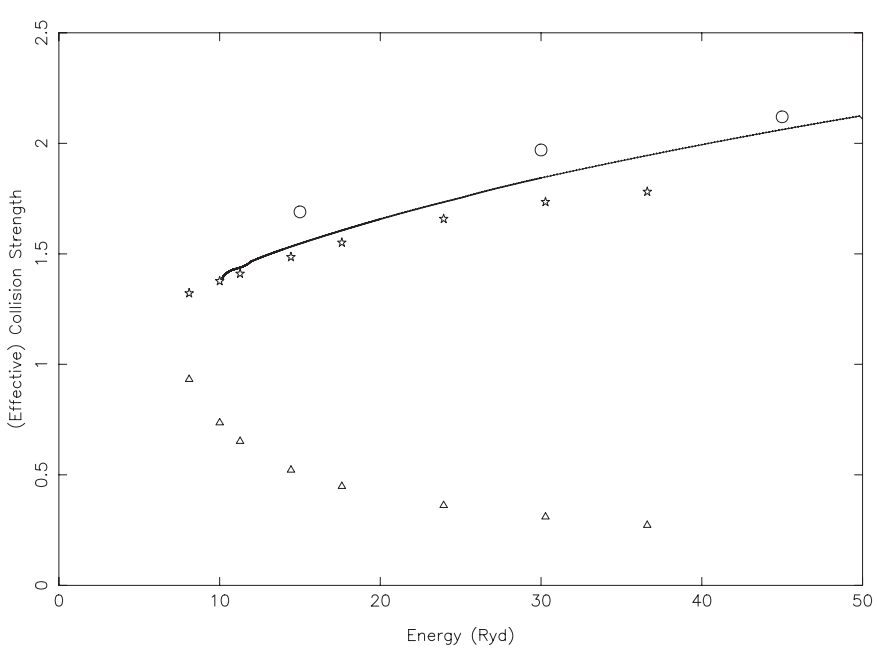

Fig. 5. Comparison of collision strengths $(\Omega)$ and effective collision strengths $(\Upsilon)$ for the $3 \mathrm{~s}^{2} 3 \mathrm{p}^{2}{ }^{3} \mathrm{P}_{1}-3 \mathrm{~s}^{2} 3 \mathrm{p} 3 \mathrm{~d}{ }^{3} \mathrm{D}_{1}^{0}(2-23)$ transition of Fe XIII, as a function of energy. Continuous curve: present $R$-matrix results of $\Omega$, circles: $\Omega$ of Gupta \& Tayal (1998), triangles: $\Upsilon$ of Gupta $\&$ Tayal (1998), and stars: present $R$-matrix results of $\Upsilon$.

while generating the wavefunctions, a large range of partial waves has been included to ensure the convergence of $\Omega$ for all transitions, and resonances have been delineated in a fine energy mesh to account for their contribution to the determination of $\Upsilon$. Apart from performing our calculations in the $j j$ coupling scheme, we have attempted to make an overall improvement over the existing results of GT. These improvements have mainly been achieved by the inclusion of: (i) a larger number of levels, i.e. 97 in comparison to the 26 of GT, (ii) a wider range of partial waves, i.e. $J \leq 39.5$ in comparison to the $J \leq 22.5$ of GT, and (iii) a higher range of energy, i.e. 120 Ryd in comparison to the 60 Ryd of GT. Additionally, results for all relevant atomic parameters are now reported for a larger number of transitions than previously available.

It is unfortunate that both sets of atomic data (of FM and GT) adopted by Landi (2002) in his detailed analysis of observational data are found to be deficient, and any agreements observed between experimental observations and theoretical determinations from their data appear to be fortuitous. Therefore, a fresh analysis of observational astrophysical data in conjunction with the present atomic data should be of considerable interest. We hope to undertake this work, employing high quality solar spectra from the SERTS missions.

Although the atomic data presented in our earlier (Aggarwal \& Keenan 2004a) and current paper for transitions in Fe XIII are perhaps the most reliable available to date, scope remains for improvement. This can mainly be achieved by improving the wavefunctions. As discussed and demonstrated in our previous paper, $\mathrm{CI}$ is very important (apart from relativistic effects) for an accurate determination of wavefunctions for this ion. In spite of observing the effect of additional CI on energy levels and radiative rates for Fe XIII, we have restricted our calculations of $\Omega$ and $\Upsilon$ to 97 levels among the above six configurations alone. This is because levels of other configurations cross over with those of the $3 \mathrm{~s} 3 \mathrm{p}^{2} 3 \mathrm{~d}$ and $3 \mathrm{~s}^{2} 3 \mathrm{~d}^{2}$ configurations, and inclusion of significantly more levels in a collisional calculation is presently beyond the limit of our available computational resources. However, it may be possible to significantly enhance the number of levels in future work. Until then the present atomic data should be of use in plasma applications.

Apart from the above mentioned scope for improvement, we do not notice any apparent deficiency in our work. Based on the comparisons made for different atomic parameters with available corresponding theoretical and experimental results, and our experience of similar work on other ions, we assess the accuracy of our energy levels to be better than 5\%. Our estimated accuracy is $\sim 20 \%$ for other parameters, namely radiative rates, collision strengths and excitation rates. However, due to the presence of a large number of resonances, especially closer to thresholds, these accuracy assessments may be insecure for results towards the lower end of the temperature range, because a slight displacement of such resonances can easily affect the listed results of $\Upsilon$ by a further $20 \%$ or so. Similarly, our results of $\Upsilon$ for transitions with higher upper levels, especially of the $3 \mathrm{~s}^{2} 3 \mathrm{~d}^{2}$ configuration, should be comparatively less accurate, because resonances arising from the higher lying levels of the neglected configurations have not been taken into account.

Acknowledgements. This work has been financed by the EPSRC and PPARC of the UK, and we wish to thank Dr. Patrick Norrington for making his code available to us prior to publication. FPK is grateful to AWE Aldermaston for the award of a William Penney Fellowship.

\section{References}

Aggarwal, K. M., \& Keenan, F. P. 2001, Phys. Scr., 64, 439

Aggarwal, K. M., \& Keenan, F. P. 2003a, MNRAS, 338, 412

Aggarwal, K. M., \& Keenan, F. P. 2003b, A\&A, 399, 799

Aggarwal, K. M., \& Keenan, F. P. 2004a, A\&A, 418, 371

Aggarwal, K. M., \& Keenan, F. P. 2004b, A\&A, 427, 763

Aggarwal, K. M., Keenan, F. P., \& Msezane, A. Z. 2003a, A\&A, 410, 349

Aggarwal, K. M., Keenan, F. P., \& Msezane, A. Z. 2003b, ApJS, 144, 169

Aggarwal, K. M., Keenan, F. P., \& Kisielius, R. 2004, A\&A, 420, 783

Brosius, J. W., Davila, J. M., Thomas, R. J., \& Monsignori Fossi, B. C. 1996, ApJS, 106, 143

Brosius, J. W., Davila, J. M., \& Thomas, R. J. 1998, ApJS, 119, 255

Brosius, J. W., Thomas, R. J., Davila, J. M., \& Landi, E. 2000, ApJ, 543,1016

Del Zanna, G., Berrington, K. A., \& Mason, H. E. 2004, A\&A, 422, 731

Dere, K. P. 1978, ApJ, 221, 1062

Dere, K. P. 1982, Sol. Phys., 77, 77

Dere, K. P., Mason, H. E., Widing, K. G., \& Bhatia, A. K. 1979, ApJS, 40, 341

Drake, J. J., Laming, J. M., \& Widing, K. G. 1995, ApJ, 443, 393

Dyall, K. G., Grant, I. P., Johnson, C. T., Parpia, F. A., \& Plummer, E. P. 1989, Comput. Phys. Commun., 55, 424

Eissner, W., Jones, M., \& Nussbaumer, H. 1974, Comput. Phys. Commun., 8, 271

Eissner, W., \& Seaton, M. J. 1972, J. Phys. B, 5, 2187

Fawcett, B. C., \& Mason, H. E. 1989, At. Data Nucl. Data Tables, 43, 245 
Feldman, U. 1981, Phys. Scr., 24, 681

Fuhrmeister, B., Schmitt, J. H. M. M., \& Wichmann, R. 2004, A\&A, 417, 701

Gupta, G.P., \& Tayal, S. S. 1998, ApJ, 506, 464

Hibbert, A. 1975, Comput. Phys. Commun., 9, 141

Jonauskas, V., Keenan, F. P., Foord, M. E., et al. 2004, A\&A, 416, 383

Keenan, F. P., Foster, V. J., Drake, J. J., Tayal, S. S., \& Widing, K. G. 1995, ApJ, 453, 906

Laming, J. M., Drake, J. J., \& Widing, K. G. 1995, ApJ, 443, 416

Landi, E. 2002, A\&A, 382, 1106

Malinovsky, M., \& Heroux, L. 1973, ApJ, 181, 1009
McKeown, K., Aggarwal, K. M., \& Keenan, F. P. 2004, Phys. Scr., 70, in press

Norrington, P. H., \& Grant, I. P. 2004, Comput. Phys. Commun., in preparation

Saraph, H. E. 1978, Comput. Phys. Commun., 3, 256

Scott, N. S., \& Taylor, K. T. 1982, Comput. Phys. Commun., 25, 347

Tayal, S. S. 2000, ApJ, 544, 575

Thomas, R. J., \& Neupert, W. M. 1994, ApJS, 91, 461

Träbert, E., Calamai, A. G., Gwinner, G., et al. 2003, J. Phys. B, 36, 1129

Young, P. R. 2004, A\&A, 417, 785 